\title{
Learning design: reflections upon the current landscape
}

\author{
Yishay Mor*a and Brock Craft $^{\mathrm{b}}$ \\ ${ }^{a}$ Institute of Educational Technology, Open University, Milton Keynes, UK; ${ }^{b}$ Institute of \\ Education, University of London, London, UK
}

(Received 12 March 2012; final version received 15 June 2012)

\begin{abstract}
The mounting wealth of open and readily available information and the accelerated evolution of social, mobile and creative technologies call for a re-conceptualisation of the role of educators: from providers of knowledge to designers of learning. This call is reverberated by the rising trend of research in learning design (LD). Addressing this, the Art and Science of Learning Design workshop brought together leading voices in the field, and provided a forum for discussing its key issues. It focused on three major themes: (1) practices, methods and methodologies, (2) tools and resources and (3) theoretical frameworks. This paper proposes a definition of LD, reviews the main contributions from the workshop, and suggests some challenges for future research.
\end{abstract}

Keywords: learning design; epistemology; design methods; design frameworks; theory development; representations

\section{Introduction}

In 1993, Alison King called for a repositioning of educators, "from sage on the stage to guide on the side". The intervening two decades has seen dramatic changes in learning, arising in no small part by innovations in technology, and recently, we are also witnessing a shift of emphasis: from distributors of knowledge to designers of learning experiences. The idea that artefacts could be devised to induce learning is not new. Indeed, Buck (1989) provides a fascinating account of learning machines designed by Archimedes, Hero of Alexandria, Quintilian - a first century Roman teacher and rhetorician, and others, to teach subjects from philosophical principles to gladiator skills. Our era is distinguished by the wealth of open and readily available information, and the accelerated evolution of social, mobile and creative technologies. These offer learners and educators unprecedented opportunities, but also entail increasingly complex challenges. Consequently, the role of educators needs to adapt from distributors of knowledge to designers for learning. Educators may still provide access to information, but now they also need to carefully craft the conditions for learners to enquire, explore, analyse, synthesise and collaboratively construct their knowledge from the variety of sources available to them. The call for such a repositioning of educators is heard from leaders in the field of technology-enhanced learning (TEL) and resonates well with the growing culture of design-based research in education. Yet, it is still struggling to find a foothold in educational practice. We contend the root causes for this discrepancy are the lack of articulation of design

*Corresponding author. Email: yishay.mor@open.ac.uk 


\section{Y. Mor and B. Craft}

practices and methods in education, the lack of a culture of teacher-as-designer among practitioners, and the shortage in tools and representations to support such practices.

In October 2011, the Art and Science of Learning Design (ASLD) workshop was convened in London, UK, to explore the tools, methods and frameworks available for practitioners and researchers invested in designing for learning, and to articulate the challenges in this evolving domain. ${ }^{1}$ The workshop adopted an unconventional design, whereby contributions were shared online beforehand, and the event itself was dedicated to synergy and synthesis. In a novel bid to make the workshop as open as possible, even to external participants, social media tools were employed to support dissemination contemporaneously with the events at the workshop. Participants were encouraged to collaboratively write and critique online, to use twitter and to contribute to workshop sessions in CloudWorks, ${ }^{2}$ a social networking site for finding, sharing and discussing learning and teaching ideas and designs.

The goal of this paper is to summarise the outputs of the workshop and to reflect on them in reference to the need to evolve the role of educators, and the barriers to this. We begin by presenting and comparing the common definitions of learning design (LD), and clarifying its links to the related but distinctly different field of instructional design (ID). We then identify some of the current issues in the field, which led to and informed the ASLD Workshop. We then present an overview of the emerging themes identified at the workshop, and guide the reader through further reading of the workshop outcomes. In the Discussion section, we examine three of the current issues and challenges raised by the workshop contributions. We explore their relevance and value to educators, content and technology developers and researchers, and finally raise some conclusions about the road ahead.

\section{Learning Design: clarifying the concept}

Design refers to the deliberate shaping of form in response to function. LD is the act of devising new practices, plans of activity, resources and tools aimed at achieving particular educational aims in a given situation. Smith and Ragan (2005) have proposed that LD might be more accurately described as Design for Learning. LD should be informed by subject knowledge, pedagogical theory, technological knowhow and practical experience. At the same time, it should also engender innovation in all these domains and support learners in their efforts and aims.

\section{Common definitions}

Koper (2006) states:

A 'learning design' is defined as the description of the teaching-learning process that takes place in a unit of learning (e.g., a course, a lesson or any other designed learning event). The key principle in learning design is that it represents the learning activities and the support activities that are performed by different persons (learners, teachers) in the context of a unit of learning.

Conole (forthcoming) defines LD as:

A methodology for enabling teachers/designers to make more informed decisions in how they go about designing learning activities and interventions, which is pedagogically informed and makes effective use of appropriate resources and technologies. 
This includes the design of resources and individual learning activities right up to curriculum-level design. A key principle is to help make the design process more explicit and shareable. Learning design as an area of research and development includes both gathering empirical evidence to understand the design process, as well as the development of a range of Learning Design resource, tools and activities.

These definitions suggest two seemingly competing approaches. Falconer, Finlay, and Fincher (2011) note that LD has two roots in TEL. The first is the construction of computer systems to orchestrate the delivery of learning resources and activities for computer-assisted learning. The second is in the need to find effective ways of sharing innovation in TEL practice, providing an aid to efficiency and professional development for teachers. Koper's definition above represents the first tradition, while Conole's is derived from the second.

The discussions at the ASLD workshop indicated a growing recognition for the need for dialogue across these two traditions, which should lead to common definitions that acknowledge LD both as a noun (activity) and a verb (the product of that activity). Such dialogue would promote the establishment of common standards - not just in the technical sense (such as the existing Integrated Management Systems-Learning Design (IMS-LD) specification), but in terms of scientific standards, best practices and measures of quality.

\section{Learning Design and Instructional Design}

In considering the current research in $\mathrm{LD}$, it is impossible to disregard the significant history of work in the domain of Instructional Design (ID). The domains of ID and LD share many broadly overlapping attributes, which can lead to some confusion among both researchers and practitioners. ${ }^{3}$ As noted by Reiser (2001), ID traces its origins to the Second World War and the need of the US military to rapidly train large numbers of people in performing technical tasks both for domestic production of war materiel and for combat. LD is more often associated with the emergence of online and TEL research in the late 1990s and 2000s. But their differences stem from more than terminology or historical origin. Most notably, they have differing theoretical backgrounds, with ID emerging from a Skinnerian perspective, strongly influenced by Tyler's ideal of behavioural objectives (Reiser 2001) while LD stems from the constructivist perspectives of Piaget and Vygotsky. This has led to a focus on learning artefacts and methods, and in designing and delivering instruction according to instructional events and their relevance to specific psychomotor learning skills. A systematic approach to task decomposition and training characterised the early methods of ID, which were later modified with the arrival of systems engineering techniques from the domain of computing and more recent research into Cognitive Load (Sweller 1994).

By contrast, the relatively recent emergence of $\mathrm{LD}$ research has seen more emphasis on the learner's context and in constructivist interpretations of the learning process, situated within an ecology of technological tools to support this. Perhaps because of its longer history, the use of ID has received greater attention in the USA than elsewhere. MacLean and Scott (2001) also observe that the level of professional support and development for LD is more developed in the USA and Canada than in the UK. Another consequence of these different historical and theoretical perspectives is that there are both gaps and overlaps in the literatures, and the research within one community, which might be particularly relevant to the other, 


\section{Y. Mor and B. Craft}

may go untapped or unnoticed. The bodies of research remain relatively siloed and cross publication is infrequent, though this is tending to diminish. Rather than take one side or the other, we feel that it is beneficial to recognise the differing traditions and the useful research from both communities by incorporating it into our thinking about LD. At the same time, our work has centred on technology-enhanced approaches to the challenging task of supporting teaching and learning, and as this is the fount from which LD has emerged, it seems a more suitable title for this collection.

\section{Current issues}

Dobozy (2011) highlights the challenges of TEL, quoting Slavin's (2002) claim that "education today is at much the same pre-scientific point as medicine was a hundred years ago", and argues that LD holds a viable potential for addressing these challenges. Yet, she contends, this potential is undermined by competing traditions and terminologies and lack of clarity, as demonstrated by Berggren et al. (2005):

The initial immersion into Learning Design gave us an experience of confusion over terms, concepts and tools. Our group constantly mixed discussions amongst conceptual points, codified specifications and multiple tools which are in various stages of development. Teachers will need to grasp these differences before a meaningful discussion can take place.

Dobozy notes that even the basic terms are contested - the field itself is called "learning design" (Dalziel 2006) "instructional design" (Chu and Kennedy 2011) "curriculum design" (Ferrell 2011) "educational design" (Goodyear and Ellis 2007), "design for learning" (Beetham and Sharpe 2007) and "design-based learning" (Wijen 2000). While it is arguable that some of these are distinct perspectives, these distinctions need to be clarified and the synergies and overlaps among the traditions need to be explored. Cameron (2010) provides a concise review of the varying perspectives on "what is learning design". Building on this, Debozy suggests a classification of three types of LD: Type $1-$ LD as a concept, Type $2-$ LD as a process and Type 3 - LD as a product. Types 2 and 3 correspond to the distinction proposed by Falconer, Finlay and Fincher (2011) above.

The ASLD workshop attempted to address the key issues of LD to meet some of its challenges. Mirroring Debozy's classification, it was organised by the three major themes: (1) practices, methods and methodologies, (2) tools and resources and (3) theoretical frameworks. The first theme is akin to Dobozy's LD Type 2 and the second to the means of production (related to Type 3). The last workshop theme centred on concepts (Type 1), to identify synergies between the other two themes. The following sections provide an overview of the contributions made by workshop participants. The full text of these contributions is available from the open, online CloudWorks repository ${ }^{2}$ of the workshop outputs. This overview is a snapshot of a particular event, and as such is, by necessity, incomplete. Yet, we believe it marks a significant point in time and highlights some of the key current debates in the field.

\section{Theme 1: practices, methods and methodologies}

The first thematic group of the workshop ${ }^{4}$ considered some contemporary trends in the practices, methods and methodologies of $\mathrm{LD}$ - from identifying and rationalising 
the stages of the LD process, to evaluating the results of design work done and interpreting what is, and is not, effective. Much of the literature in LD describes the representations or the products of design work, but not the process itself.

Dimitriadis, Prieto and Villagrá-Sobrin emphasise the importance of the contextual logistics of learning, when designs and design patterns are enacted in the classroom. They use several examples to illustrate how high- and low-level formalisations impact on teachers and learners.

Another formalisation, the IMS-LD approach, has attracted noteworthy attention within the LD community and has gained a lot of support from researchers since its introduction in 2003. However, its uptake has remained relatively slow, given this level of interest. Responding to this observation, Griffiths, Goddard, and Wang describe a study comprising interviews of practitioners and leading participants in the IMS-LD community which attempts to explain the limitations to its adoption.

Ronen-Fuhrman and Kali describe a study of graduate students in education the very people who will put contemporary research in LD into practice. They show how the students' use of an epistemological model to aid them in designing learning in TEL modules closes gaps between theoretical and applied knowledge. This work also informs the refinement of the innovative Design Principles Database which could be useful not only to students but to seasoned teachers.

Masterman notes the importance of evidence in assessing the effectiveness of the design process, using a case study to describe a method of analysing LD software. She also highlights the important consequences for LD more broadly.

\section{Theme 2: tools and resources}

In addition to the difficulty of developing design practices and methods, an ancillary challenge is that there are few tools to support the LD process itself. Many other design-focused disciplines have seen the emergence of significant software supports for their work. Architects and engineers have their CAD tools, Graphic Designers and 3D animators can choose from a range of creative suites, and there are even packages for designing performative activities such as theatrical lighting. Yet, to support the complex process of LD there are comparatively few tools to choose from. The second thematic group of the workshop, along with a hands-on session, explored some of the most recent and promising of them. ${ }^{5}$

As research in LD has evolved, so have a modest number of software systems and platforms to support design activities. These implement methods of LD at various levels of learning activity and provide support for sharing work with others. Familiar representations are important for giving teachers new ways to engage with technology to enhance LD. Helen Walmsley illustrates this principle by presenting a simple pedagogic template in the form of a Word document. She shows how this can be extremely effective for creating curriculum and tool-focused e-Learning, at this micro-level of design work, where planning individual learning activities or sessions occurs. An emphasis on sharing and co-edition are also the basis for a case study in the use of LdShake, described by Hernández-Leo et al. (2011). Their focus on socialnetwork-oriented work and sharing across teams and institutions also illustrates how innovations in LD can affect larger initiatives across schools and communities. Ryberg et al. describe a method that emphasises collaboration in the design process. They show how this can help in creating activities for networked learning. 


\section{Y. Mor and B. Craft}

Several tools were offered for hands-on experience, and are available on CloudWorks. As a contrasting view on the challenges raised for IMS-LD in the first thematic group, Katasmani and Retalis present CADMOS, a system that achieves the challenge of providing support with IMS-LD compliance. Their study shows how the right tool for the right purpose can support teachers meaningfully in their work by addressing specific challenges such as the "separation of concerns". Derntl presents another system, OpenGLM, which supports the first two levels of IMS-LD design, but without the need to be an expert in the framework. It provides a set of visual representations and simple interactions to aid practitioners in designing and sharing IMS-LD-based designs. Effective representations and ease-of-use are intimately intertwined. As noted extensively in Botturi and Stubbs (2008), significant challenges are raised by the difficulty of meaningfully expressing such representations. Brasher et al. have created a tool for just this purpose-CompendiumLD, and reflect upon what they have learned in using and refining it. They show how the challenges of representation will likely become more acute as technology-supported LDs become richer and more complex.

Learning design systems can equally operate at more strategic level to support thinking about both learning and the required resources to support it. Laurillard and Masterman describe such a system, the Learning Designer, that supports such decisions with an Artificial Intelligence (AI)-driven recommender engine, and facilitates sharing and reuse.

Rounding out this panoply of software, Emin and Pernin describe both a conceptual framework for LD (ISiS), and a tool (ScenEdit) that implements it. Their work straddles two thematic stands and highlights how tight integration of a theory-driven conceptual framework, can be effectively manifested in software.

\section{Theme 3: theories and frameworks}

Theory generation can be achieved both from findings of research aimed at theory building and from reflection on practitioner experiences. Such theories can support the development of conceptual frameworks of knowledge to support the LD process. The third thematic group of the ASLD workshop explored some of the emergent theories and knowledge frameworks that are influencing the epistemology of LD.

Prieto, Dimitriadis, and Villagrá-Sobrin introduce the notion of "atomic patterns" and use it to propose a representation for LDs (especially collaborative LDs using multiple Information Communications Technology (ICT) tools), which tries to depict how the activities are actually enacted by the teacher in the classroom. Fleshing out an applied perspective, Burgos illustrates some of the practical challenges of implementing IMS-LD, providing evidence from several learning scenarios and a case study. The challenges to adoption of IMS-LD serve to illustrate both the importance and the difficulty of providing a unified foundation on which to support learning designers and practitioners. Examining how this and other kinds of TEL innovation work can impact on everyday teachers, McKinney takes a look at the broader picture by highlighting how the gaps between theory and practice can be bridged. Both fine-grained issues in classrooms and more systemic issues must be addressed. She outlines significant methodological considerations that should be considered by designers and researchers in this context. Cook further explores some considerations researchers must be sensitive to, by showing how research can be scaled up for large techno-pedagogical designs. Two projects illustrate the attendant 
challenges. They shed light on means of grappling with the difficult problems of building systems for larger audiences of learners in mobile and informal learning contexts - a key area of theoretical development that is just beginning to see significant attention. New frameworks will have to be developed to support work in this area. Persico and Pozzi call for just this kind of research, framework development. They conclude this thematic strand with an analysis that provides a multi-dimensional framework drawing together a number of approaches and tools for design of learning. Their analysis of four key areas of research in LD, namely, representation, abstraction, pedagogic approach and types of end users identifies essential areas for further investigation. This conclusion to the thematic strand on Theories and Frameworks shines a light on the potential areas for fruitful continued research and development and provides an epistemological capstone to the thematic strand on Methods and Tools.

\section{Discussion}

The ASLD workshop is indicative of the growing awareness and vibrant community of researchers and practitioners shaping the field of LD. On the one hand, the field is maturing, with the articulation of theoretical and methodological frameworks, the availability of a wide choice of tools, and the buildup of a cannon of literature. On the other hand, several challenges are emerging as clear directions for future work.

The first is the standardisation of a comprehensive representational infrastructure. By this, we mean human-readable and writable, textual and graphical (and perhaps dynamic) forms of describing LD at multiple levels of abstraction. One metaphor that surfaced repeatedly at the ASLD workshop was that of musical notation. Musical notation enables complex, expressive, dynamic, time-based content to be captured accurately and succinctly, yet expressively. Moreover, the symbolic, formalised abstraction of the content does not impede interpretation and reproduction. On the contrary, capturing the "essence" of a musical work formally facilitates the creative expression of the composer, whilst leaving room for interpretive reproduction of musicians. In the genre of Jazz for example, the music of Miles Davis can be interpreted in myriad ways by many musicians, without losing its essential nature. Teachers have frequently related their desire to record the essence of their practice (at various levels of detail and with many kinds of activities), whilst not sacrificing their ability to be creative, due to limitations of the means of capture. This analogy breaks down, if pushed too far. In the end, one would like to be able to assert that a particular teaching method or approach leads to better learning experiences among students, and some formative or summative assessment metric is inevitably involved in demonstrating this. Music, a largely aesthetic endeavour, is less germane to this kind of evaluation. However, the impact of notation on dissemination, sharing and indeed creative expression of music would be hard to understate. Another useful analogy is the language of architectural drawings. An architect's design process is scaffolded by a progression through a series of graphical and other articulations of their ideas. These representations afford a discussion of the design objectives and the means chosen to address them. They eventually require the interpretation of craftsmen in order to be implemented as physical buildings, yet any professional can assess whether a certain construction matches the design prescribed in the drawings. The field of LD enjoys an impressive array of textual, graphical and computational representations of practice and resources. However, it still lacks the canonical "score" 


\section{Y. Mor and B. Craft}

or "drawing" of music and architecture. In order for educators to effectively orchestrate learning within this landscape, they need to perceive themselves, and indeed to be perceived by society, as techno-pedagogical designers. A design attitude should be reflected in the production of new resources, as well as in effective configuration and customisation of existing ones. The design paradigm has established itself in TEL research. Yet, for it to attain its full desired impact, it needs to develop a common language and make this language accessible to the widest possible audience. Such a language, and the related media of interaction, should allow experts and novices to extract design knowledge from experience, articulate it in a coherent manner, connect, combine and manipulate it, and use it to resolve new challenges.

This leads us naturally to the second challenge: a common language of LD needs to be supported by appropriate tools and community spaces, which will streamline the process of constructing, validating and using design knowledge, making it open, accessible and transparent. It cannot be a uniform, centralised entity. It must allow for a diversity of discourse by establishing a set of open protocols and standards over which an open process of massively collaborative knowledge building can thrive. This process needs to be embedded in the culture of the professional community. Again, recent years have witnessed the flourishing of an impressive arsenal of LD tools. Yet, no single tool can address the requirements of all practitioners in all situations. Nor can a single tool can provide a "round trip" solution, which must support the full cycle from inception, through challenge definition, conceptualisation, elaboration, enactment, evaluation and reflection and back to remodelling. Thus, the question is: how do we create a platform for open, live, malleable, dynamic representations of design knowledge in TEL, supporting collaborative processes of design for learning, learning to design and learning by design, and including the broadest community possible in these processes?

A common language of LD and a comprehensive platform to support it are necessary but not sufficient conditions for the emergence of a professional culture of LD. An open platform for LD might promote the emergence of a new culture of educational practice, in which expertise is rapidly and effectively shared, critiqued and aggregated. It will provide for the wide proliferation of cost-effective and robust educational practices, making effective use of technological advances as they appear. However, such a culture will not be instigated simply by the existence of the right tools and representations. The existing LD community needs to engage in a massive project of professional development, driving a new perception of educational profession, as a rigorous creative practice of perpetual innovation. The principles underlying the LD approach, the practices reifying those principles, and the methodological framework binding those together need to be made explicit and communicated to the widest audience possible.

Finally, the uncharted links and dimensions of a design approach to educational practice need to be exploded. Other design disciplines emphasise their creative and aesthetic qualities. How are these reflected in the domain of LD? Should we promote them, and how? Can we evaluate the creative and aesthetic qualities of a particular LD process or artefact? On the other hand, design approaches have recently gained prominence in educational research. Should we, and can we, forge links between design-based research and research-inspired practice? Several studies (RonenFuhrmann, Kali, and Hoadley 2008; Voogt et al. 2011) demonstrate the value of engaging in design for teachers' professional development. This is no surprise, if we 
acknowledge educators' continuous development as a learning process, and consider learning-by-design as a powerful pedagogical framework. Mitch Resnick (2007) calls for re-conceptualising education to promote the creative society. In order to do that, we need to re-conceptualise teaching as a creative practice. With this in mind, we propose a view of LD as a grounded rigorous creative process of perpetual educational innovation: grounded in a well-defined concrete context of practice, rigorous in its attention to scientific evidence and pedagogical theory, and creative in its approach to generating new solutions to educational challenges.

\section{Conclusion}

We believe that the great deal of enthusiastic research in LD will continue to be fruitful for the key recipients of our industrious efforts: other researchers, teachers and not least, learners. Indeed many of us within the research community have been or continue to be teachers and learners, ourselves. These communities come in many shapes and from diverse, rich traditions and cultures around the world. Yet, they face the common challenges of mutual collaboration, sharing and support in the complex social and increasingly, socio-technical process that is twenty-first century learning. The work represented here is emblematic of the aspirations to meeting this challenge and confirms that we are heading in the right direction, together.

\section{Acknowledgements}

The ASLD workshop, and the writing of this paper, where supported by the Learning Design Grid theme team of the STELLAR network of excellence (www.ld-grid.org).

\section{Notes}

1. http://www.ld-grid.org/workshops/ASLD11

2. http://cloudworks.ac.uk/cloudscape/view/2349

3. For an interesting overview of the lively discussions surrounding this, see "LD vs. ID", a Cloudworks discussion thread at http://cloudworks.ac.uk/cloud/view/2536

4. http://cloudworks.ac.uk/cloud/view/5790

5. http://cloudworks.ac.uk/cloud/view/5793 and http://cloudworks.ac.uk/cloud/view/5841

\section{References}

Beetham, H. \& Sharpe, R. (2007) Rethinking Pedagogy for a Digital Age, Routledge, New York, NY, 10001.

Berggren, A., et al. (2005) 'Practical and pedagogical issues for teacher adoption of IMS learning design standards in moodle LMS', Journal of Interactive Media in Education, vol. 2005 , no. 1 , pp. $1-24$.

Botturi, L. \& Stubbs, S. T. (2008) Handbook of Visual Languages for Instructional Design. Theories and Practices, Information Science Reference, Hershey, PA.

Buck, G. H. (1989) 'Teaching machines and teaching AIDS in the ancient world', McGill Journal of Education/Revue des sciences de l'éducation de McGill, vol. 24, no. 1, pp. 31-54.

Cameron, L. (2010) 'How learning design can illuminate teaching practice', The Future of Learning Design Conference, Wollongong, Australia, December 2009, [online] Available at: http://ro.uow.edu.au/fld/09/Program/3/

Chu, S. \& Kennedy, D. (2011) 'Using online collaborative tools for groups to co-construct knowledge', Online Information Review, vol. 35, no. 4, pp. 581-597.

Conole, G. (forthcoming) Designing for learning in an open world, Springer, New York. 


\section{Y. Mor and B. Craft}

Dalziel, J. R. (2006) 'Lessons from LAMS for IMS learning design', Sixth International Conference on Advanced Learning Technologies, IEEE, Kerkrade, Netherlands, July 2006, pp. 1101-1102.

Dobozy, E. (2011) 'Typologies of learning design and the introduction of a "LD-Type 2" case example', eLearning Papers, Vol. 27, no. 27, pp. 1-11. [online] Available at: http:// elearningeuropa.info/sites/default/files/asset/In-depth_27_1.pdf

Falconer, I., Finlay, J. \& Fincher, S. (2011) 'Representing Practice: Practice models, patterns, bundles', Learning Media and Technology, vol. 36, no. 2, pp. 101-127.

Ferrell, G. (2011) Transforming Curriculum Design - Transforming Institutions, Briefing paper, Joint Information Systems Committee (JISC), [online] Available at: http://www.jisc.ac.uk/ publications/briefingpapers/2011/bpcurriculumdesign.aspx

Goodyear, P. \& Ellis, R. (2007) 'Students' interpretations of learning tasks: Implications for educational design', Proceedings of the ASCILITE 2007 conference, Singapore, pp. 339346.

Hernández-Leo, D. et al. (2011) 'LdShake: Learning design solutions sharing and co-edition', Computers \& Education, vol. 57, no. 4, pp. 2249-2260.

Koper, R. (2006) 'Current research in learning design', Educational Technology \& Society, vol. 9, no. 1, pp. 13-22.

Maclean, P. \& Scott, B. (2007) 'Learning design: Requirements, practice and prospects', Campus-Wide Information Systems, vol. 24, no. 3, pp. 187-98.

Reiser, R. (2001) 'A history of instructional design and technology: Part II: A history of instructional design', Educational Technology Research and Development, vol. 49, no. 1, pp. $57-67$.

Resnick, M. (2007) 'Sowing the seeds for a more creative society', Learning and Leading with Technology, vol. 35, no. 4, pp. 18-22.

Ronen-Fuhrmann, T., Kali, Y. \& Hoadley, C. (2008) 'Helping education students understand learning through designing', Educational Technology, vol. 48, no. 2, pp. 26-33.

Slavin, R. E. (2002) 'Evidence-based education policies: transforming educational practice and research', Educational Researcher, vol. 31, no. 7, pp. 15-21.

Smith, P. L. \& Ragan, T. J. (2005) Instructional Design, Wiley, Hoboken, NJ, USA.

Sweller, J. (1994) 'Cognitive load theory, learning difficulty, and instructional design', Learning and Instruction, vol. 4, no. 4, pp. 295-312.

Voogt, J., et al. (2011) 'Teacher learning in collaborative curriculum design', Teaching and Teacher Education, vol. 27, no. 8, pp. 1235-1244.

Wijen, W. (2000) Towards design-based learning, Technische Universiteit, Eindhoven, NL, OGO brochure, No 2. Educational Service Centre, [online] Available at: http://w3.tue.nl/ fileadmin/stu/stu_oo/doc/OGO_brochure_1_EN.pdf 